\title{
idvf: Iterative Inversion of Deformation Vector Field with Adaptive Bi-residual Feedback Control
}

\section{Alexandros-Stavros lliopoulos ${ }^{1}$, Abhishek Dubey ${ }^{1}$, and Xiaobai Sun ${ }^{1}$}

1 Department of Computer Science, Duke University, Durham, NC 27708, USA

DOI: $10.21105 /$ joss. 01076

\section{Software}

- Review ¿

- Repository 七

- Archive c

Submitted: 02 November 2018 Published: 29 March 2019

\section{License}

Authors of papers retain copyright and release the work under a Creative Commons Attribution 4.0 International License (CC-BY).

\section{Summary}

We provide a package for fast and accurate inversion of a deformation vector field (DVF). A DVF, also known as flow or dense motion field, describes a non-linear mapping between a study image and a reference image. DVFs are fundamental to several medical image analysis and computer vision applications. The inverse DVF is often needed together with the forward DVF to enable back-and-forth mappings, and composite mappings among multiple images. Relevant applications include 4D image reconstruction, anatomical atlas generation, dose accumulation estimation in adaptive radiotherapy, simultaneous deformable registration, and symmetric registration completion. To our knowledge, no other package is available for DVF inversion with guaranteed convergence.

We use an iterative inversion procedure and employ adaptive bi-residual feedback control to achieve global convergence and local acceleration (Dubey, 2018; Dubey, Iliopoulos, Sun, Yin, \& Ren, 2018). The inverse DVF estimate and the forward DVF must meet the inverse consistency (IC) condition. We define two IC residuals, which measure the inconsistency between two DVFs in the study and reference domains. The residuals, which are computationally available, are related to the unknown inversion error. We use them as feedback in a two-phase iteration. In the first phase, we modulate the study-domain IC residual with adaptive feedback control for guaranteed global convergence, under certain mild conditions (Dubey et al., 2018). Once the error is made sufficiently small, we switch to phase two where we use the reference-domain IC residual and achieve locally quadratic convergence rate. Phase transition and integration is enabled by a multi-resolution scheme (Dubey, 2018).

A pre-release version of idvf has been used in scientific publications to demonstrate the inversion algorithm framework and analysis (Dubey et al., 2018) and potential applications (Dubey, 2018). The idvf package is implemented in MATLAB. Its source code is archived with Zenodo (Iliopoulos, Dubey, \& Sun, 2018).

\section{References}

Dubey, A. (2018). Symmetric completion of deformable registration via bi-residual inversion (PhD thesis). Duke University, Durham, NC, USA.

Dubey, A., Iliopoulos, A.-S., Sun, X., Yin, F.-F., \& Ren, L. (2018). Iterative inversion of deformation vector fields with feedback control. Medical Physics, 45(7), 3147-3160. doi:10.1002/mp.12962

Iliopoulos, A.-S., Dubey, A., \& Sun, X. (2018). idvf: Iterative inversion of deformation vector field with adaptive bi-residual feedback control. doi:10.5281/zenodo.1476602 